\title{
De la recherche de l'information aux pratiques informationnelles
}

From Information Seeking to Information Practices

\section{Stéphane Chaudiron et Madjid Ihadjadene}

\section{CpenEdition}

\section{Journals}

Édition électronique

URL : http://journals.openedition.org/edc/2257

DOI : $10.4000 /$ edc. 2257

ISSN : 2101-0366

Éditeur

Université de Lille

Édition imprimée

Date de publication : 1 décembre 2010

Pagination : 13-30

ISBN : 978-2-917562-04-8

ISSN : $1270-6841$

Référence électronique

Stéphane Chaudiron et Madjid Ihadjadene, « De la recherche de l'information aux pratiques

informationnelles », Études de communication [En ligne], 35 | 2010, mis en ligne le 01 décembre 2012, consulté le 10 décembre 2020. URL : http://journals.openedition.org/edc/2257 ; DOI : https://doi.org/ $10.4000 /$ edc. 2257

Ce document a été généré automatiquement le 10 décembre 2020.

(c) Tous droits réservés 


\title{
De la recherche de l'information aux pratiques informationnelles
}

\author{
From Information Seeking to Information Practices
}

Stéphane Chaudiron et Madjid Ihadjadene

1 La question des pratiques informationnelles est à la confluence de questionnements scientifiques dont les origines sont multiples. Cette question s'inscrit d'abord dans l'évolution des modalités d'analyse des dispositifs d'accès à l'information; d'abord centrées sur l'aspect technique des dispositifs, les analyses se sont ensuite progressivement intéressées à la manière dont ils étaient effectivement utilisés. Elle s'inscrit ensuite dans l'évolution et des méthodes utilisés pour étudier ces usages, issues de disciplines différentes et, au sein même des disciplines, de traditions épistémologiques et culturelles différentes. Elle trouve son origine enfin dans les interrogations de nature plus conceptuelle qui concernent en particulier les notions de "dispositif ", d'« interaction ", d'« usage ", d'« appropriation » et bien sûr de " pratiques ».

2 L'analyse des dispositifs d'accès à l'information a longtemps été abordée à travers le prisme du paradigme dit « système " (Ellis, 1992) dont les fondements sont empruntés aux sciences physiques. Dans cette approche, l'information et les systèmes qui la gèrent $y$ sont présentés comme des éléments extérieurs à l'usager. Les premières études portant sur les dispositifs d'accès à l'information et plus précisément sur les systèmes de recherche d'information (SRI), datent des années 1950 et ont d'abord privilégié une approche technique, essentiellement dans le cadre de campagnes d'évaluation comparative (Chaudiron, 2001). Se démarquant progressivement de cette approche techno-centrée, différentes courants, réunis sous le terme générique de paradigme " usager ", se sont ensuite attachés à étudier les "information behaviors " en prenant progressivement en considération l'usager dans ses différentes dimensions, cognitive, psychologique, affective voire communicationnelle. Plus récemment, l'émergence de ce que A. Halavais nomme la "search engine society » (Halavais, 2009) a conduit un troisième courant de recherches à intégrer dans son analyse le rôle de l'environnement socioéconomique dans l'accès et la maîtrise de l'information, notamment les dimensions 
linguistiques et culturelles, la question des inégalités face à l'information (en particulier la fracture numérique), l'impact des stratégies de référencement ou la publicité en ligne...

Questionner les pratiques informationnelles nécessite d'étudier les modalités d'inscription de ce concept dans les différentes champs disciplinaires qui en font usage, au premier rang desquels les sciences de l'information (library and information science) et les sciences de la communication (media and communication studies). Mais il s'agit également d'intégrer dans la réflexion les mutations techniques des dispositifs de production, de recherche, de partage et de diffusion de l'information qui induisent des transformations organisationnelles en déplaçant par exemple les frontières professionnelles dans le cycle du traitement de l'information. Plusieurs exemples illustrent parfaitement ces mutations : l'émergence du data journalism place ainsi les journalistes au cœur des fonctions documentaires avec la nécessité de maîtriser le Web sémantique, la fouille de données (dataminig) ou la sémiologie visuelle. Un autre exemple est celui du Web 2.0 et des réseaux sociaux (Facebook, Twitter...) qui remettent en cause la fonction traditionnelle de gatekeeper du journaliste en donnant la possibilité aux internautes d'être des producteurs et des éditeurs de contenu. Un dernier exemple concerne l'évolution du métier de veilleur et plus particulièrement sa fonction de diffusion de l'information; bien que depuis longtemps intégrée dans la veille, cette fonction évolue grâce aux nouveaux outils (Content management systems, réseaux sociaux, agrégateurs de presse, logiciels de filtrage et d'analyse de contenu...) et amplifie ainsi le rôle de médiation informationnelle.

4 Ces quelques exemples montrent que nous assistons à l'émergence d'un nouvel écosystème informationnel qui décloisonne et modifie les pratiques informationnelles en banalisant l'accès aux dispositifs de médiation, en réduisant la distance entre expert/ professionnel et novice/amateur, et en déplaçant les frontières professionnelles traditionnelles.

5 Dans cet article, nous tentons de cerner le concept de " pratique informationnelle ». Dans la première partie, nous essayons de le clarifier en le situant par rapport à d'autres termes voisins tels que " usage et 'comportement' "; puis, dans une deuxième partie, nous essayons d'en montrer la genèse en le situant dans l'évolution des courants scientifiques depuis la fin des années 1950. Enfin, dans la dernière partie, nous proposons de renouveler les modèles informationnels à l'aide des apports des sciences de la communication et nous concluons en nous interrogeant sur la pertinence du concept de " pratique info-communicationnelle».

\section{De l'usage aux pratiques informationnelles : essais de clarification conceptuelle}

6 L'étude des dispositifs d'accès à l'information, et plus généralement des dispositifs techniques de médiation de l'information, pose un problème terminologique et conceptuel. Comme nous l'avons montré (Ihadjadene et Chaudiron, 2008), les chercheurs s'intéressant à ces objets d'études utilisent une terminologie à la fois diverse et polysémique. On constate ainsi que les termes tels que "utilisation ", " usage ", " recherche ", " besoin ", " pratiques " sont employés en références à des cadres théoriques différents et correspondent à des réalités diverses. Ainsi, ces travaux, qui sont très majoritairement d'origine nord-américaines, britanniques et, mais dans une moindre 
mesure, scandinaves, peuvent être regroupés sous différents labels tels que les besoins d'information et d'usage (information need), les usagers (users studies), les modalités d'accès et d'usage de l'information (information seeking and use), les comportements informationnels (information behavior ou human information behavior) et, plus récemment, les pratiques informationnelles (information practices).

7 Un premier problème terminologique est la traduction en français de termes qui s'inscrivent dans des espaces notionnels différents. Ainsi, en raison de la connotation "behavioriste ", beaucoup plus négativement marquée en français qu'en anglais, le terme «information behavior» est fréquemment traduit par «pratiques informationnelles ». Un ouvrage de l'ASIST (American society for information science and technology) qui présente 72 modèles différents des «comportements " informationnels n'hésite en effet pas à porter le titre de Theories of Information Behavior (Fischer, 2005). Ces auteurs intègrent dans ce courant de recherche aussi bien les travaux sur les stratégies et les tactiques de recherche d'information (information searching behavior) que sur les modalités d'accès et d'usage de l'information (information seeking and use). Pour éviter de recourir au terme " comportement informationnel ", nous avons suggéré d'utiliser celui de " pratiques informationnelles », mais dans une acception plus large, puisqu'il désigne la manière dont un ensemble de dispositifs, de sources formelles ou non, d'outils, de compétences cognitives sont effectivement mobilisés, par un individu ou un groupe d'individus, dans les différentes situations de production, de recherche, d'organisation, de traitement, d'usage, de partage et de communication de l'information. Nous englobons dans ce terme de " pratique » aussi bien les comportements, les représentations que les attitudes informationnelles de l'humain (individuel ou collectif) associés à ces situations (Ihadjadene et Chaudiron, 2008). Cette acception intègre donc, mais excède, la définition traditionnellement donnée à la notion de « information practice » dans le contexte de la recherche d'information par des auteurs tels que Pamela McKenzie (2003) qui a élaboré un modèle des pratiques informationnelles visant à rendre compte de la recherche d'information " dans la vie de tous les jours » (everyday-life information seeking), ou Sanna Talja et Preben Hansen qui la définissent comme les " practices of information seeking, retrieval, filtering and synthesis » (Talja et Hansen, 2005, 113).

8 Sur le plan conceptuel, il convient également de distinguer les notions de " pratique » et " usage ». Alors que le terme "utilisation " renvoie donc à la manière dont un utilisateur particulier, dans sa singularité propre, découvre, appréhende et manipule un dispositif (comme un moteur de recherche par exemple), la notion d'usage a un sens plus large. Selon Philippe Breton et Serge Proulx (2006), il est employé dans une perspective sociologique pour décrire le cadre social large qui englobe les interactions entre les humains et les machines. Comme le précise Rémy Rieffel (2005), c'est au début des années 1980 que les premiers travaux portant sur les usages "orientés-usager » des TIC apparaissent. Les chercheurs parlent alors "d'usagers " et non "d'utilisateurs ». On assiste ainsi à une modification de perspective et à un glissement de cadre théorique dans la mesure où les travaux sur l'usager portent non plus sur la seule dimension cognitive de l'interaction personne-système mais sur ses dimensions sociale et symbolique. Comme le souligne Joëlle Le Marec $(2001,146)$, «le cumul des recherches menées [...] a permis de dégager progressivement la notion d'usage de sa référence implicite à la maîtrise du fonctionnement des objets techniques ".

9 Néanmoins, les usages ne peuvent pas être pensés indépendamment des interactions entre des individus, socialement et cognitivement situés, et des dispositifs qui sont 
organisées dans le cadre d'un contrat de communication qui, tel un contrat de lecture, fixe une série de règles a priori indiquant les possibles et les impossibles. En ce sens, les usages sont envisagés comme l'expression d'un processus constitué d'interactions complexes mettant en relation un individu et un dispositif qui peut être, ou non, un artefact technique. Une caractéristique de la dimension d'usage est donc sa (relative) stabilité, à la fois diachronique et synchronique. Diachronique, dans la mesure où l'usage est ancré dans un cadre socio-technique de référence (Flichy, 1995) et synchronique parce qu'il est observable collectivement à un moment donné. C'est ainsi que l'on peut parler des usages de l'internet (Guichard, 2001) ou des usages avancés des téléphones mobiles (Licoppe et Zouinar, 2009). Nous rejoignons en ce sens Jacques Perriault (1989) selon lequel l'usage est une "utilisation stabilisée d'un objet, d'un outil, pour obtenir un effet ».

Pour Josiane Jouët (1993), la notion de pratique est plus élaborée que celle d'usage car elle prend en compte l'utilisation des techniques, mais également les comportements, les attitudes, les représentations de ceux qui y ont recours. Contrairement à elle, il ne nous semble pas qu'il y ait un enchâssement de l'une dans l'autre, mais qu'il s'agit de deux notions qui permettent d'appréhender la réalité selon deux angles différents et complémentaires. La distinction que nous proposons suggère de réserver le terme d'usage pour désigner les travaux portant sur les dispositifs, techniques ou non, et leurs interactions avec les usagers ; le terme de pratique sera réservé pour caractériser les approches centrées sur le « comportement composite " à l'œuvre dans les différentes sphères, informationnelles, culturelles, journalistiques, etc. L'observation des pratiques, qu'elles soient individuelles ou collectives, nécessite alors d'adopter une approche de l'action envisagée comme un processus en tension entre les savoirs mobilisables, les compétences immédiates, les habitus, les arts de faire, les désirs d'agir, etc.

On parlera donc des usages des moteurs de recherche ou des téléphones portables, des usages de l'internet ou des usages des bibliothèques numériques pour désigner la façon dont on utilise le dispositif en tenant compte du cadre socio-professionnel, culturel, politique, économique... Les usages des dispositifs techniques s'inscrivent en ce sens dans différents contextes, professionnel, privé, familial... À l'inverse, nous parlerons de pratiques pour désigner les études qui sont centrées sur l'humain (individuel ou collectif) et qui analyse ses modalités d'action (ou d'inaction), ses représentations, ses attitudes. Le terme de pratique est habituellement utilisé dans les champs professionnels mais peut l'être également dans des contextes autres tels que le grand public. On peut ainsi s'intéresser aux pratiques quotidiennes de recherche d'information pour résoudre des problèmes de la vie courante non reliés au contexte de travail (comme les pages jaunes, l'horaire des trains, l'information médicale...), domaine que les anglo-saxons nomment ELIS (everyday-life information seeking), développé notamment par Pamela McKenzie (2003) ou Reijo Savolainen (1995 et 2008).

On parlera alors de pratiques informationnelles pour désigner la manière dont l'ensemble de dispositifs, des sources, des outils, des compétences cognitives sont effectivement mobilisés dans les différentes situations de production, de recherche, traitement de l'information. 


\section{De la recherche à l'usage de l'information}

13 À l'inverse de l'approche orientée système, le paradigme " usager » considère donc que l'attention doit être davantage portée sur les besoins réels de l'usager et son environnement. Ce paradigme s'inscrit dans une perspective constructiviste dans laquelle la démarche de repérage et de traitement de l'information par l'usager ne peut faire abstraction d'un ensemble de facteurs tant individuels que situationnels. Les deux approches relèvent de communautés différentes même si plusieurs chercheurs tentent depuis la fin des années 1990, d'établir des ponts entre les deux approches. Saracevic (1997), Ingwersen et Järvelin (2005), Belkin (2008) ont proposé d'associer les deux paradigmes en une théorie unifiée de la recherche d'information (unified theory of information seeking and retrieval). Cette théorie, ou tout au moins ce programme de travail, se construit sur un certain nombre de considérations mettant l'accent sur le rôle du contexte, de l'émotion, de l'incertitude et des interactions entre le système et l'usager dans l'élaboration des processus cognitifs. Dans cette perspective, de nombreux chercheurs se sont penchés sur la modélisation des pratiques de recherche d'information.

Un premier courant regroupe les travaux qui concernent directement les interactions de l'usager avec le SRI en privilégiant certains facteurs ou dimensions comme la définition et l'analyse du besoin informationnel, notamment (Taylor, 1968) (Belkin, 1980) ou (Dervin, 1992), la prise en compte des tâche dans l'interaction, (Vakkari, 2003), (Marchionini, 1995), (Byström-Jãrvelin, 1995), la description des stratégies et des tactiques (Bates, 1989) ou la modélisation de l'interaction, (Spink, 1997), (Bates, 1989), (Saracevic, 1996), (Belkin, 1995). D'autres auteurs comme Peter Ingwersen (1996) ou JeanFrançois Rouet et André Tricot (1998) ont privilégié la dimension cognitive.

De très nombreux travaux, principalement d'origine nord-américaine, britannique et scandinave, ont ainsi été développés depuis les années 1980 pour expliciter les pratiques d'accès à l'information, terme qui traduit ici l'expression anglaise de « information seeking behavior » et qui englobe aussi bien le besoin que l'usage de l'information. Ce courant de recherche vise à mieux préciser les processus d'accès aux sources formelles (Ellis, 1997), la dimension affective de l'accès à l'information (Khulthau, 1993), le contexte professionnel (Leckie et al., 1996), (Thivant et Bouzidi, 2005), la description des situations (Cheuk Wai-Yi, 1998) et enfin l'usage de l'information, (Taylor, 1991), (Kari, 2010). Dans cette perspective, différents terrains sont étudiées : celui de la formation (collégiens, lycéens, étudiants), de l'activité professionnelle (médecins, juristes, chercheurs, ingénieurs...) ou celui de la vie quotidienne comme les pratiques d'accès des consommateurs, des citoyens, des militants...

16 Les travaux sur les pratiques d'accès à l'information négligent souvent d'analyser l'impact de l'information recherchée. Néanmoins, un auteur comme R. Taylor (1991) a proposé un modèle permettant d'identifier les différentes catégories d'utilisation de l'information. Il suggère en particulier que le contexte de l'utilisation de l'information est un facteur déterminant pour comprendre la diversité des comportements informationnels. Il propose donc un modèle appelé Information Use Environment qui définit cet environnement comme un ensemble d'éléments qui exercent une influence sur la circulation de l'information entre individus ou groupes d'individus et qui, de ce fait, constituent des critères permettant de juger de la valeur de l'information dans un contexte précis. Taylor identifie trois types principaux d'environnements d'utilisation de l'information : 
l'environnement géographique (le pays), l'environnement organisationnel (l'entreprise) et enfin l'environnement social/intellectuel/culturel, représenté par des groupes de personnes partageant des valeurs et des intérêts communs. En s'appuyant sur ces travaux, H. Rosenbaum (1996) propose une modélisation du contexte d'usage de l'information dans les organisations. Il présente un modèle dans lequel les règles techniques et procédurales jouent un rôle dominant dans la définition des pratiques informationnelles et qui évoluent en fonction du contexte technologique et humain. Le second élément structurel concerne les ressources utilisées, en particulier les dispositifs technologiques ainsi que les produits informationnels. Les deux dernières composantes du modèle de $\mathrm{H}$. Rosenbaum ont trait aux problèmes et à la résolution de problèmes dans l'environnement d'utilisation de l'information.

Toutefois, il manque des travaux pour appréhender le rôle de l'information dans le changement des comportements des individus ou d'une organisation. Quelle est l'influence des sources d'information sur l'action des individus ? Comment transformer les informations collectées en prise de décision, qu'elle soit économique, sociale, ou culturelle ? Or, si des progrès sont réalisés dans la connaissance des pratiques d'accès à l'information, les travaux portant sur la réception et l'utilisation de l'information en contexte sont relativement peu nombreux. Les rares travaux d'organismes comme l'UNESCO (Correra et al., 1997) sur la diffusion de l'information en milieu rural ou médical nous renseignent d'abord sur l'insuffisance d'une analyse essentiellement centrée sur l'accès et la recherche, et sur l'inadéquation des modèles pour appréhender les autres aspects des pratiques informationnelles.

Un dernier ensemble de travaux, qui s'inscrit dans une approche que l'on qualifiera d'holistique, ne s'intéresse plus à l'utilisateur comme individu isolé face à un dispositif mais comme un usager situé dans son contexte social, culturel et linguistique. L'impact des dynamiques interpersonnelles et sociales est alors intégré dans l'analyse des pratiques informationnelles. Plusieurs auteurs se sont attachés à intégrer les variables sociales dans leur modélisation des pratiques.

19 Tom Wilson fut ainsi l'un des premiers à proposer en 1981 (Wilson, 1981) un modèle fondé sur l'hypothèse que les besoins informationnels exprimés par les usagers des systèmes de recherche d'information étaient issus de besoins plus fondamentaux, d'ordre physiologique, cognitif ou affectif, liés au contexte personnel ou social de l'individu et à son environnement (politique, économique, technologique...). Il suggérait que les obstacles à surmonter pour accéder à l'information souhaitée provenaient de ce contexte ou de cet environnement. Un second modèle, plus général, élargit le premier en substituant la notion d'« intervening variables » à celle de " barrière " pour souligner qu'il existe des facteurs extérieurs à l'individu qui facilitent ou au contraire freinent l'accès à l'information (Wilson, 1996).

De son côté, Elfreda Chatman (1999) a souligné l'importance de l'environnement et du capital social dans les pratiques informationnelles. L'observation au milieu des années 1980 des minorités noires et économiquement pauvres aux États-Unis, a mené Chatman à intégrer dans sa réflexion la théorie de l'aliénation inspirée des travaux de Merton, de Durkheim et de Weber. Comme les normes sociales traduisent les valeurs et les idéaux dominants de la société ou du groupe, elle estime qu'elles régissant aussi le comportement informationnel. Integrated Model ») et généraliste où l'on retrouve à la fois la manifestation du besoin 
d'information, la recherche de l'information et l'utilisation de l'information. Pour C. Choo, il est nécessaire de considérer l'information non comme un objet, mais plutôt comme le résultat d'une construction subjective ; à ce niveau, les facteurs cognitifs, affectifs et situationnels jouent un rôle déterminant mais sont à considérer dans le cadre de l'organisation.

Un dernier exemple peut être celui de R. Savolainen (2008) qui montre également que les normes et les règles du groupe, de la profession ou de l'organisation ont aussi un impact sur le traitement et l'utilisation de l'information par les individus. Le monde social, de l'entreprise par exemple, est ainsi constitué d'acquis dont l'intégration est implicite dans les actions et les intentionnalités pratiques. La connaissance n'est plus individuelle mais située socialement. Pour comprendre la complexité des pratiques informationnelles, il est donc important de décrire le champ dans lequel évolue l'usager c'est-à-dire son environnement, la position qu'il occupe au sein de ce champ, l'ensemble des compétences qu'il mobilise et les mécanismes d'interprétation de l'information.

Ces différents exemples illustrent la pluralité d'approches qui caractérise l'observation des pratiques informationnelles. Le cumul des recherches menées au cas par cas depuis plus de quarante ans, par delà la variété extrême des terrains, des méthodes et des théories, a permis de construire un champ de recherche en le dégageant de l'emprise techniciste. Le recueil régulier de données variées sur les interactions des usagers avec les dispositifs d'accès à l'information (notamment avec les moteurs de recherche) et leur exploitation quantitative et qualitative ont également permis de cumuler des résultats significatifs aboutissant à une amélioration de ces dispositifs. Toutefois, plusieurs interrogations demeurent. D'abord, l'analyse de ces modèles révèle une grande diversité dans les niveaux d'analyse : les différences individuelles, l'activité, les groupes professionnels, le style cognitif, l'organisation, le secteur d'activité, la tâche, le dispositif d'accès, l'interface homme-machine, etc. Il est difficile dans ces conditions d'établir une taxonomie fiable.

Par ailleurs, ces modèles ne font pas intervenir la dimension réflexive de l'usager qui se traduit pas des activités métacognitives ; ils supposent pour la plupart une interaction continue avec le système d'information et ne rendent pas compte des ruptures en cours de processus, occasionnées par exemple soit par une interaction entre pairs, soit par la consultation d'une autre source (expert, base de données, etc.). En conséquence, le caractère opérationnel des modèles n'est pas toujours établi : la modélisation devrait permettre de déterminer avec précision les variables qui interviennent dans le comportement ainsi que le rôle de ces différentes variables mais c'est rarement le cas. Se pose ainsi la question de la pondération des variables et des mesures afférentes. La variation des niveaux d'analyse pose des problèmes d'interprétation des résultats qui engage un glissement et une re-formalisation des concepts qui n'ont en principe leur pertinence qu'à un niveau particulier. Cette complexité n'a néanmoins pas entravé la diffusion des modèles informationnels qui ont, de ce fait, beaucoup influencé le développement de la culture numérique ainsi que la maitrise de l'information en améliorant les dispositifs de formations (information literacy). On peut toutefois s'interroger à propos de la pertinence des modèles théoriques qui visent à modéliser le comportement informationnel des usagers et des tâches qu'ils accomplissent. Il est en effet souvent difficile de faire un lien opérationnel entre le cadre et les finalités méthodologiques des études et les cadres théoriques portés par les modèles de l'usage qui sont proposés en sciences de l'information. Il n'est pas certain qu'il soit encore possible 
d'élaborer une théorie générale avec une logique explicative unique de l'ensemble des pratiques informationnelles. Une telle démarche conduit souvent, pour rester fidèle au cadre interprétatif, à avancer des analyses réductrices de nombre de comportements, d'attitudes, de stratégies de recherche.

\section{Vers une analyse des pratiques « info- communicationnelles »?}

Les études en sciences de la communication ont décrit et analysé depuis presque trente ans les réactions des destinataires des programmes face aux offres qui leur étaient adressées. Ces travaux (études de réception, sociologie de la culture et des pratiques culturelles, sociologie des usages, analyse des médiations, théorie critique, etc...) constituent un apport majeur pour une meilleure compréhension et modélisations des pratiques informationnelles. Mais, du fait que les deux champs scientifiques, sciences de l'information et sciences de la communication sont distincts dans les pays anglo-saxons et encore souvent déconnectés en France, la majorité des travaux sur les pratiques informationnelles, principalement issus de la library and information science, ne font pas référence à ces courants théoriques. Ainsi, pendant longtemps, les études sur l'information ont été pensées en excluant de prendre en compte les phénomènes de communication qui l'accompagnent (Fondin, 2006). C'est probablement l'une des raisons pour lesquelles la majorité des modèles informationnels minorent les activités de partage et de communication de l'information et privilégient l'activité d'accès et de recherche.

Pourtant, les mutations techniques des dispositifs de production, de recherche, de partage et de diffusion de l'information qui induisent des transformations organisationnelles, en favorisant l'émergence d'un nouvel écosystème informationnel, constituent un terrain privilégié pour renouveler les approches sur les pratiques informationnelles. Ces nouveaux dispositifs (Web 2.0, réseaux sociaux, CMS, logiciels de filtrage...) banalisent les modalités de production de l'information et fusionnent les fonctionnalités de recherche et d'édition. En ce sens, les dispositifs d'accès à l'information actuels (notamment les moteurs de recherche) présentent les caractéristiques d'un nouveau média (plutôt d'un web-media). Jean-Michel Salaun (2006) et Alexander Halavais (2009) soulignent que le monde du web emprunte le modèle économique à la radiotélévision car il est fondé sur une économie de l'attention mais qu'il emprunte aussi au modèle de la bibliothèque par le partage et la mise en commun des connaissances. Ces dispositifs sont des médias c'est-à-dire des objets qui, pour reprendre Yves Jeanneret (2009), ne font pas que s'associer à du social, mais qui en produisent via notamment des médiations symboliques et représentationnelles. De même, la numérisation accrue des activités humaines, marchandes ou non, et l'évolution des métiers rendent progressivement inopérante l'opposition entre l'information grand public produite par les organes de presse et l'information scientifique et technique (Polity, 2000). La jonction avec les approches communicationnelles peut mettre en évidence des enjeux liés à la médiation des savoirs, à la gestion de l'identité numérique et à la question des pouvoirs.

Dépassant leur statut de simples récepteurs, les usagers jouent désormais un rôle actif dans la production, le classement, l'évaluation de l'information. Les individus, mais aussi les organisations, mettent aussi en œuvre des stratégies ou des politiques pour faciliter l'échange d'information et sa communication via des plateformes d'intermédiation 
(Intranet, réseaux sociaux, etc.). Les individus sont aussi des acteurs dans la production de leur réputation numérique, de la valorisation de leurs images, de l'impact de leurs participation à des réseaux sociaux sur leur vie privée ou professionnelles bref de la gestion de leurs identités numériques (Georges, 2010).

Une piste de recherche qui nous semble stimulante et mobilisatrice est d'étudier les pratiques informationnelles sous l'angle de la médiation. En effet, dans une institution culturelle ou dans une organisation (notamment pour la gestion de leur contenu informationnel), le choix des normes et standards, les politiques d'indexation et de classement, les opérations d'inventaire et de constitution de collection ne sont pas neutres mais sont médiés par des logiques sociales. Selon Bernadette Dufrene (2007), ces opérations ne sont pas « indépendantes du cadre dans lesquels elles s'élaborent ; leur sens est relatif à des conditions d'énonciation propres à des types d'institutions et à des situations historiques ". L'intérêt d'une approche sous l'angle de la médiation est d'amorcer une critique des conditions dans lesquelles les textes, les données, les dispositifs sont produits afin de mieux cerner leur interprétation. (Jeanneret, 2009). Chaque médiation conditionne les pratiques même si, selon Jean Davallon (1999, 78), « le guidage de la réception reste probabiliste ».

Enfin, nous pensons que d'autres considérations, négligées auparavant, interviennent désormais dans toute étude sur les usages des dispositifs d'accès à l'information : la question éthique (avec la traçabilité des usagers, l'échange, la conservation et la commercialisation des données personnelles), la question économique (publicité en ligne, marchandisation accrue $d u$ lien social, monétisation du contexte via le géoréférencement, etc.), la question identitaire (création de communautés en ligne, redéfinition des identités, etc.), la question politique (la censure dans l'indexation des sites par les moteurs, le respect de la diversité culturelle, etc.), les enjeux juridiques (la mise en ligne d'œuvres protégées, l'accès à des contenus illicites via des moteurs spécialisés...). La mise en évidence de ces enjeux socio-politiques au niveau de l'individu, d'une organisation ou de la société permet de refléter des rapports de pouvoir issus de la généralisation des dispositifs d'accès et de diffusion de l'information.

Une piste de recherche est d'enrichir et de renouveler les modèles informationnels à l'aune de ces apports. Il s'agirait alors pour nous de proposer une approche infocommunicationnelle des dispositifs d'accès et de diffusion de l'information.

\section{BIBLIOGRAPHIE}

Bates, M. J., (1989), The Design of Browsing and Berrypicking Techniques for the Online Search Interface, in Online Review, Vol. 13, october, pp. 407-424.

Belkin, N., (1980), Anomalous states of knowledge as a basis for information retrieval, in The Canadian Journal of Information Science, $\mathrm{n}^{\circ}$ 5, pp. 133-143. 
Belkin, N., Cool, C., Stein, A. et Thiel, U., (1995), Cases, scripts and information-seeking strategies : On the design of interactive information retrieval systems, in Expert Systems with Applications, Vol. 9, n 3, pp. 379-395.

Belkin, N., (2008), Some(what) grand challenges for information retrieval, in SIGIR Forum, Vol. $42, \mathrm{n}^{\circ} 1$, pp. 47-54.

Breton, P. et Proulx, S., (2006), L'explosion de la communication. Introduction aux théories et aux pratiques de la communication, Paris, La Découverte, 384 p.

Byström, K. et Järvelin, K., (1995), Task complexity affects information seeking and use, in Information Processing \& Management, Vol. 31, $\mathrm{n}^{\circ}$ 2, pp. 191-213.

Chatman, E. A., (1999), A theory of life in the round, in Journal of the American Society for Information science, Vol. 50, $\mathrm{n}^{\circ}$ 3, pp. 207-217.

Chaudiron, S., (2001), L'Évaluation des systèmes de traitement de l'information textuelle : vers un changement de paradigme, thèse d'habilitation à diriger des recherches, Université de Paris 10.

Chaudiron, S. et Ihadjadène, M., (2002), Quelle place pour l'usager dans l'évaluation des SRI, in Actes du Colloque international « Recherches récentes en sciences de l'information : convergences et dynamiques » (sous la dir. de Viviane Couzinet et Gérard Regimbeau), Paris, ADBS, pp. 211-231.

Chaudiron, S., (2004), L'évaluation des systèmes de recherche d'informations, in Les systèmes de recherche d'informations : modèles conceptuels (sous la dir. de M. Ihadjadene), Paris, Hermès, pp. $185-207$.

Chaudiron, S., (2004), La place de l'usager dans l'évaluation des systèmes de recherche d'informations, in Évaluation des systèmes de traitement de l'information (sous la dir. de S. Chaudiron), Paris, Hermès, pp. 287-310.

Chaudiron, S. et Ihadjadène, M., (2004), Évaluer les systèmes de recherche d'informations : nouveaux modèles de l'utilisateur, in Hermès, $n^{\circ}$ 39, pp. 170-178.

Cheuk Wai-Yi, B., (1998), Modelling the information seeking and use process in the workplace : employing sense-making approach, in Information Research, Vol. 4, $\mathrm{n}^{\circ} \quad$ 2. Disponible sur : http://informationr.net/ir/4-2/isic/cheuk.html (Page cconsultée le 28 septembre 2010).

Choo, C. W., Detlor, B. et Turnbull, D., (2000), Web Work : Information Seeking and Knowledge Work on the World Wide Web, Dordrecht, Kluwer Academic publishers, 236 p.

Correra, F. A., Ndiaye, D. et al., (1997), Rural Information Provision in Developing Countries Measuring Performance and Impact, prepared for UNESCO, Paris, UNESCO, 116 p.

Davallon, J., (1999), L'exposition à l'œuvre : stratégies de communication et médiation symbolique, Paris, L'Harmattan, 382 p.

Davenport, T. H., (1997), Information Ecology : Mastering the Information and Knowledge Environmen, New York, Oxford University Press.

Dervin, B., (1992), From the mind's eye of the user : The sense-making qualitative- quantitative methodology, in Jack D. Glazier and Ronald R. Powell (éds.) Qualitative Research in Information Management, Englewood, Libraries Unlimited, pp. 68-70.

Dufrene, B., (2007), Intérêts d'une approche sociohistorique des questions de médiation culturelle, in Actes des journées d'étude " Quelles approches de la médiation culturelle ?", Lille, 9-10 décembre 2004,pp. 237-244. 
Ellis, D. et Haugan, M., (1997), Modelling the information seeking patterns of engineers and research scientists in an industrial environment, Journal of Documentation, Vol. 53, $\mathrm{n}^{\circ} 4$, pp. 384-403.

Ellis, D., (1992), The Physical and cognitive paradigm in information retrieval research, in Journal of Documentation, Vol. 48, $\mathrm{n}^{\circ} 1$, pp. 45-64.

Fisher, K., Erdelez, S. et McKechnie, L. (éds.), (2005), Theories of Information Behavior, ASIST, Information Today Inc., 431 p.

Flichy, P., (1995), L'innovation technique. Récents développements en sciences sociales. Vers une nouvelle théorie de l'innovation, Paris, La Découverte, 250 p.

Fondin, H., (2006), La science de l'information ou le poids de l'histoire, in Les enjeux de l'information et de la communication. Disponible sur : http://w3.u-grenoble3.fr/ les_enjeux/2005/Fondin/home.html (Page consultée le 02 septembre 2010).

Georges, F., (2010), Identités virtuelles. Les profils utilisateur du Web 2.0, Ed. Questions théoriques, $201 \mathrm{p}$.

Guichard, E. (éd.), (2001), Comprendre les usages de l'internet, Paris, Éditions Rue d'Ulm, 261 p.

Halavais, A., (2009), Search Engine Society, Cambridge, Polity Press, 232 p.

Ihadjadene, M. et Chaudiron, S., (2008), L'Étude des dispositifs d'accès à l'information : approches croisées, in Problématiques émergentes dans les sciences de l'information (sous la dir. de F. Papy), Paris, Hermès-Lavoisier, pp. 183-207.

Ingwersen, P., (1996), Cognitive perspectives of information retrieval interaction : elements of a cognitive IR theory, in Journal of Documentation, Vol. 52, $\mathrm{n}^{\circ}$ 1, pp. 3-50.

Ingwersen, P. et Järvelin, K., (2005), The Turn : Integration of Information Seeking and Retrieval in Context, Heidelberg, Springer, 448 p.

Jeanneret, Y., (2009), La relation entre médiation et usage dans les recherches en informationcommunication en France, in Electronic Journal Commun. Information and Innovation in Health, Vol. 3, $\mathrm{n}^{\circ}$ 3. Disponible sur : http://www.reciis.cict.fiocruz.br/index.php/reciis/article/ view/276/320 (Page consultée le 2 septembre 2010).

Jouet, J., (1993), Pratiques de communication et figures de la médiation, in Réseaux, $\mathrm{n}^{\circ}$ 60, Paris, Hermès, pp. 99-120.

Kari, J., (2010), Diversity in the conceptions of information use, in Information Research, Vol. 15, $\mathrm{n}^{\circ}$ 3. Disponible sur : http://informationr.net/ir/15-3/colis7/colis709.html (Page consultée le 28 septembre 2010).

Kuhlthau, C., (1993), Seeking meaning : A process approach to library and information services, Norwood, Ablex, 199 p.

Leckie, G. J., Pettigrew, K. E. et Sylvain, C., (1996), Modeling the information seeking ofprofessionionals : a general model derived from research on engineers, health care professionals, and lawyers, in Library Quarterly, Vol. 66, $\mathrm{n}^{\circ}$ 2, pp. 161-193.

Le Marec, J., (2001), L'analyse des usages en construction : quelques points de méthode, in Comprendre les usages de l'internet, E. Guichard (sous la dir. de), Paris, Éditions Rue d'Ulm, pp. 146-155.

Licoppe, C. et Zouinar, M., (2009), Les usages avancés du téléphone mobile, in Réseaux, $\mathrm{n}^{\circ} 156$, La Découverte, 292 p. 
Marchionini, G., (1995), Information seeking in electronic environments, New York, University Press, $224 \mathrm{p}$.

McKenzie, P., (2003), A model of information practices in account of everyday life information seeking, in Journal of Documentation, Vol. 59, $\mathrm{n}^{\circ} 1$, pp. 19-40.

Perriault, J., (1989), La logique de l'usage. Essai sur les machines à communiquer, Paris, Flammarion, $254 \mathrm{p}$.

Polity, Y., (2000), Information I versus Information II, Communication au groupe de travail "Théories et Pratiques Scientifiques » de la SFSIC, 27 octobre 2000. Disponible sur : http:// www.iut2.upmf-grenoble.fr/RI3/Information.htm (Page consultée le 2 septembre 2010).

Talja, S. et Hansen, P., (2005), Information sharing, in New Directions in Human Information Behavior, (sous la dir. de A. Spink et C. Coles), Berlin, Springer, pp. 113-134.

Rieffel, R., (2005), Sociologie des médias, Ellipses Marketing, Paris, 223 p.

Rosenbaum, H., (1996), Structure and action : Towards a new concept of the information use environment, in Proceedings of ASIS' 96 Annual Conference : https://scholarworks.iu.edu/ dspace/bitstream/handle/2022/1804/wp96-04B.html?sequence=1 (Page consultée le 28 septembre 2010).

Rouet, J.-F. et Tricot, A., (1998), Chercher de l'information dans un hypertexte : vers un modèle des processus cognitifs, in Les hypermédias : approches cognitives et ergonomiques, (sous la dir. de A. Tricot et J.-F. Rouet), Paris, Hermès, pp. 57-74.

Saracevic, T., (1996), Modeling interaction in Information Retrieval (IR) : A review and proposal, in Proceedings of the Annual Meeting of the American Society for Information Science, Vol. 33 pp. 3-9.

Saracevic, T., (1997), Users lost : Reflections on the past, future, and limits of information science, in Proceedings of ACM-SIGIR, pp. 16-27.

Savolainen, R., (1995), Everyday-life information seeking : approaching information seeking in the context of way of life, in Library \& Information Science Research, Vol. 17, $\mathrm{n}^{\circ}$ 3, pp. 259-294.

Savolainen, R., (2008), Everyday Information Practices. A Social Phenomenological Perspective, Plymouth, The Scarecrow Press Inc., 232 p.

Spink, A., (1997), Study of interactive feedback during mediated information retrieval, in Journal of the American Society for Information Science, Vol. 48, $n^{\circ}$ 5, pp. 382-394.

Taylor, R., (1968), Question-negociation and information seeking in libraries, in College and research libraries, $n^{\circ} 29$, pp. 178-194.

Taylor, R., (1991), Information use environments, in Progress in Communication Sciences, B. Dervin et M. Voigt (éds.), Norwood, Ablex Publishing, Vol. 10, pp. 217-255.

Thivant, E. et Bouzidi, L., (2005), Les pratiques d'accès à l'information : le cas des concepteurs de produits de placements financiers, in Revue électronique suisse de science de l'information, $\mathrm{n}$ - 2, pp. 7-34.

Vakkari, P., (2003), Task-based information searching, in B. Cronin et D. Shaw (éds.), Annual Review of Information Science and Technology, Information Today, Medford, pp. 413-464.

Wilson, T. D., (1981), On user studies and information needs, in Journal of Documentation, Vol. 37, n 1, pp. 3-15. 
Wilson, T. D., (1996), Information behaviour : an interdisciplinary perspective. A report to the British Library Research and Innovation Centre, London, British Library Research and Innovation Centre : http://informationr.net/tdw/publ/infbehav/chap7.html (Page consultée le 27 septembre 2010).

Wilson, T. D., (2000), Human information behavior, in Informing Science, Vol. 3, $\mathrm{n}^{\circ} 2$, pp. $49-55$.

\section{RÉSUMÉS}

Cet article introduit le concept de «pratique informationnelle ». Après l'avoir défini par rapport aux concepts d' « usage » et de « comportement », nous proposons une analyse de son évolution à travers les différents champs scientifiques tels que l'informatique sociale, les sciences de l'information et la sociologie des usages qui contribué à sa définition. L'article conclut sur les apports possibles des sciences de la communication pour le renouvellement des modèles informationnels.

This article introduces the concept of information practice. After analysing it, in relation to the notions of « information seeking and use » and « information behaviour », it offers a reviewing of its evolution through the different scientific trends, such as social informatics, library and information science and sociology of use, which have contributed to its elaboration. Lastly, the paper discusses the possible contribution of communication science in renewing information models.

\section{INDEX}

Mots-clés : pratique informationnelle, usage, recherche d'informations, comportement, sociologie des usages

Keywords : information practice, information behaviour, information seeking and use, communication science

\section{AUTEURS}

\section{STÉPHANE CHAUDIRON}

Laboratoire GERiiCO - Université de Lille 3

Stéphane Chaudiron est professeur en Sciences de l'information et de la communication à l'université de Lille 3. Il est membre du laboratoire GERiico dont il est actuellement le directeur. Ses travaux portent sur : l'évaluation des systèmes de traitement avancé de l'information et leurs usages, l'analyse des pratiques informationnelles, l'organisation des connaissances et l'épistémologie des SIC. Adresse électronique : stephane.chaudiron@univ-lille3.fr.

\section{MADJID IHADJADENE}

Laboratoire Paragraphe - Université de Paris VIII

Madjid Ihadjadene est professeur en Sciences de l'information et de la communication à

l'université de Paris 8. Il est membre du laboratoire Paragraphe et responsable de l'équipe INDEX (INformation Documents et contEXtes). Ses domaines de recherche sont: l'épistémologie des SIC, l'analyse des pratiques informationnelles, les bibliothèques numériques, l'usage des dispositifs de 
médiation et d'accès à l'information, l'organisation des connaissances. Adresse électronique : madjid.ihadjadene@univ-paris8.fr. 